\title{
Using Attribute and Attitude Assessment for Bidding in Automated Auctions
}

\begin{abstract}
An automated auction is an efficient market institution for realworld trading of commodities. This paper presents a novel fuzzy bidding strategy(FAA-Bid), which employs assessments of multiple attributes of items as well as agents' attitude on bidding item to procure an item in automated auction. The assessment of attributes adapts the fuzzy sets technique to handle uncertainty of the bidding process as well use heuristic rules to determine attitude of bidding agents in simulated auctions to procure goods. The overall assessment is used to determine a price range based on current bid, which finally selects the best one as the new bid.
\end{abstract}

\section{INTRODUCTION}

In electronic market places, auctions are widely recognised as efficient mechanisms to allocate goods and resources to the entities that value them most highly. Intelligent software agents $[1,3,9,10]$ provide a powerful tool to address complex problems of dynamic pricing in automated auctions. The agents can use different auction mechanisms (e.g. English, Dutch, Vickery etc.) for procurement of goods or reaching agreement between agents. The agent makes decisions on behalf of consumer and endeavours to guarantee the delivery of item according to the buyer's preferences. In these auctions buyers are faced with difficult task of deciding amount to bid in order to get the desired item matching their preferences. For this reason, the formalisation of bidding mechanism has received a great deal of attention from the agent community for the past decade. These software agents should be smart enough to bargain a favourable deal for the user. In order to be called an intelligent agent, the software must satisfy several criteria like autonomy, temporal continuity, communication and cooperation. To this end, a number of researchers $[14,15,17,19,21]$ have reported different frameworks that help an autonomous agent to tackle the problem of bidding in auctions. Currently, no single implementation satisfies all the criteria, but there are several promising results for bargaining intelligent agents.

In this paper, a fuzzy bidding strategy (FAA-Bid) is designed in an automated auction based on the dual assessment of multiple attributes of items as well as agents's attitude on bidding item. To

Permission to make digital or hard copies of all or part of this work for personal or classroom use is granted without fee provided that copies are not made or distributed for profit or commercial advantage and that copies bear this notice and the full citation on the first page. To copy otherwise, to republish, to post on servers or to redistribute to lists, requires prior specific permission and/or a fee.

Copyright 200X ACM X-XXXXX-XX-X/XX/XX ...\$10.00. quantify attitudes and to deal with uncertainty of attribute assessment fuzzy sets technique is applied in the presented strategy. The basic procedure of the strategy is shown in Figure 1. The remainder of the paper is organized as below. First, the detail of the presented strategy is illustrated. Then, a simple experiment is conducted. Related work and conclusion are discussed finally.

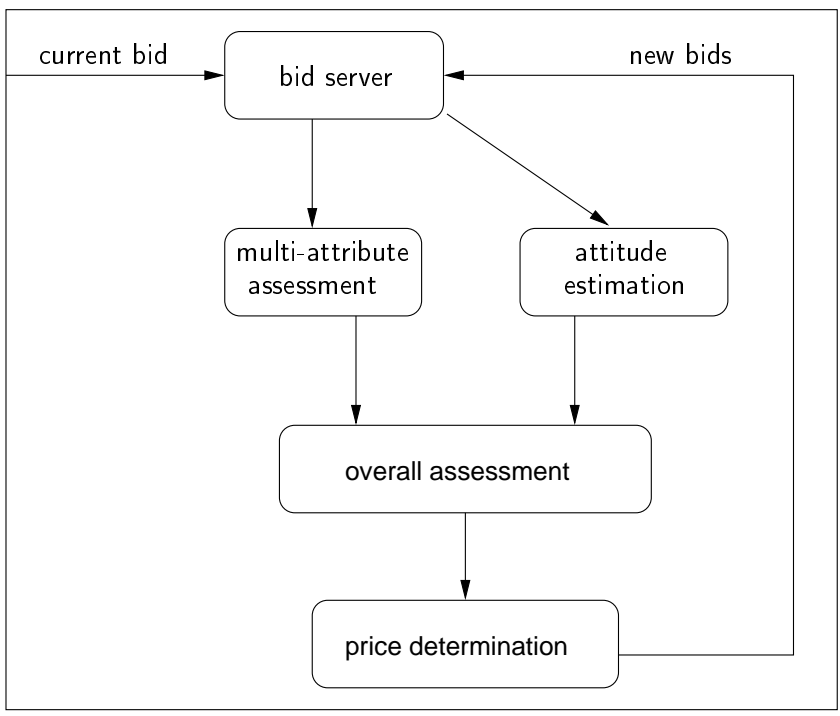

Figure 1: A Fuzzy Bidding Strategy (FAA-Bid) Model.

\section{RELATED WORKS}

There have been several approaches from computer scientists for developing intelligent software methods and protocols for automated negotiation. In particular, the first trading agent competition (TAC) was held in Boston in July 2000 [12]. TAC agents acted as simulated travel agents and had to procure goods for their clients in different types of auctions, bidding against autonomous agents. Priest et al. [20] proposed an algorithm design for agents that participate in multiple simultaneous English auctions. The algorithm proposes a coordination mechanism to be used in environment where all the auctions terminate simultaneously, and a learning method to tackle auctions that terminate at different times. Byde et al. [3] presented another decision theoretic framework that an autonomous agent can use to bid effectively across multiple auctions with various protocols. The framework uses an approximation function that provides an estimate of the expected utility of participating in the set of future auctions and it can be employed to purchase single or multiple items. Anthony et al. [1] proposed an 
approach for agents to bid for a single item in English, Dutch, and Vickrey auctions. The agent decides what to bid based on four parameters: i) the remaining time; ii) the number of remaining auctions; iii) the desire for bargain; and iv) the desperateness of the agent. The overall strategy is to combine these four tactics using a set of relative weights provided by the user. In an extension to this model [19], a genetic algorithm is used to search the effective strategies so that an agent can behave appropriately according to its assessment of its prevailing circumstances. The machine learning techniques [21] are also used to obtain a model of the price dynamics based on the past data (e.g., the data in the seeding round) to predict the closing prices of the hotels in the future. It also uses mixed-integer linear programming to find the optimal allocation of the goods.

Considering the existed uncertainty in auctions, the fuzzy techniques are used to manage an agent's interactions. Faratin et al. [7] used fuzzy similarity to compute trade offs among multiple attributes during bilateral negotiations in order to find a win-win solution for both parties. Kowalcyzk and Bui $[13,14]$ modeled the multi-issue negotiation process as a fuzzy constraint satisfaction problem. Their approach performs negotiation on individual solutions one at a time. During negotiation, an agent evaluates the offers, relaxes the preferences, constraints and makes counter-offers to find an agreement for both parties. Luo et al. $[15,16]$ developed a fuzzy constraint based framework for bilateral multi-issue negotiations in semi-competitive trading environments. The framework is expressed via two knowledge models, one for the seller agent and one for the buyer agent. The seller agent's domain knowledge consists of its multi-dimensional representation of products or services it offers. The buyer agent's domain knowledge consists of the buyer's requirement/preference model (a prioritized fuzzy constraint problem) and buyer's profile model (fuzzy truth propositions).The buyer and seller agents exchange offers and counteroffers with additional constraints revealed or existing constraints being relaxed. Finally, a solution is found if there is one.He et al. [10] proposed the fuzzy logic strategy, which was the first time that fuzzy sets and fuzzy reasoning were introduced into the heuristic rules for agents. A fuzzy logic seller or buyer calculates an ask or a bid by considering the relationship among the outstanding bid, the outstanding ask, and the reference price which was the median of the ordered price history. It uses fuzzy reasoning techniques to predict closing price of auctions, fuzzy recognition to assess the degree of competitiveness in the prevailing market context, anf fuzzy set technique to control bidding behavior. Ma and Leung [17] developed and evaluated the adaptive attitude strategy that exploits both the short term and long term attitudes, and utilizes a set of heuristic rules in bid determination.

Different researchers have also provided alternatives to fuzzy reasoning for coping with the uncertainties in bidding. For example, the possibility-based approach [18] has been used to perform multi-agent reasoning under uncertainty for bilateral negotiations where uncertainties due to the lack of knowledge about other agents' behaviors are modeled by possibility distributions. The Bayesian learning method has also been used to model multiissue negotiation in a sequential decision making model. In [26], a Bayesian network is used to update the knowledge and belief each agent has about the environment and other agents, and offers and counter-offers between agents during bilateral negotiations are generated based on Bayesian probabilities.

\section{A FUZZY BIDDING STRATEGY (FAA- BID)}

\subsection{Basic Scenario}

In this section, we will present an automated bidding strategy using fuzzy sets and attitudes. Our strategy is discussed based on the following scenario.

1) Suppose a travel agent wants to book tickets for some clients. These clients have different preference and requirements on the possible tickets. Assume that six factors are concerned in this situation, i.e. ticket price $\left(c_{0}\right.$, e.g. from $\left.\$ 800-\$ 2000\right)$, depart time $\left(c_{1}\right.$, e.g. 18:00 PM, Wednesday), arrival time ( $c_{2}$, e.g. 10:00 AM, Friday), number of stops ( $c_{3}$, e.g. at most 3 stops), seat positions $\left(c_{4}\right.$, e.g. near Window, Aisle, etc), and travel season ( $c_{5}$, e.g. off-peak season).

2) Suppose the identified perspective of an agent is summarized as below:

- The agent prefers to a cheaper ticket and agrees to that the cheaper the better.

- The agent prefers to travel at the weekend rather than at working day.

- The agent prefers to no stop travel.

- The agent prefers to aisle seat then window seat.

- The agent prefers to travel during off-peak season rather than peak season.

- The agent thinks the flight price is the most important factor, secondly the travel season, and other factors are of same importance.

3) Based on the client's perspective, the agent evaluates a flight ticket using several terms (such as "very bad", "bad", "slightly bad", "acceptable", "fairly good", "good", "very good", etc.).

Using this scenario, an agent is required to bid for a flight ticket based on the its attitude.

\subsection{FAA-Bid Overview}

In an automated auction, an agent's bidding activity is influenced mainly by two aspects, namely, 1) the attributes of goods and 2) the agent's attitude. Any agent prefers to make a bid for a quality goods by adopting an appropriate bidding strategy. Considering the existence of uncertainty in a real auction situation, this paper focuses on how to make bid by using the agent's personal perspective.

To make a bid for a unit of goods, the agent should balance between his/her assessment on the goods and his/her attitude to win an auction. Generally speaking, an agent has stronger attitude to make bid for a quality goods rather than a lower one. The attitude is mainly based on the assessment on the goods. Moreover, an agent's attitude is also influenced by the bids because price is the unique factor through which agents negotiate till make a deal. To win an auction, an agent must balance among the price (bid), assessment on the goods and attitude to win a bid.

Roughly speaking, the bidding procedure runs as follows:

- Firstly, evaluation on each related attributes is determined.

- Then these evaluations are aggregated to form an overall assessment on the goods.

- Next, the attitude of the agent is determined.

- Overall assessment is conducted.

- Finally, a new bid is determined. 
Since in real situation uncertainty exists ubiquitously in expressing assessments, attitude as well as their relationships with price, this paper uses fuzzy-set-based method to process uncertainty in assessment and attitude. First of all, this paper uses a satisfactory degree measure as the common universe of assessment, i.e., an assessment is treated as a fuzzy set on the satisfactory degree. Secondly, an attitude is expressed as a fuzzy set on the set of assessments, i.e., the assessment set is the universe of attitude.

In the following sections, details of the strategy is illustrated.

\subsection{Attribute Evaluation}

Attribute evaluation includes two kinds of process. The first one is individual attribute assessment, and the second one is assessment aggregation. To implement attribute evaluation, three issues are concerned, i.e., attribute weights (relative importance) determination, assessment expression, and assessment aggregation.

\subsubsection{Weights Determination}

Weights indicate different preference of an agent on each identified factor. In this paper, we use the Analytic Hierarchy Process (AHP) method [22] to determine the weight for each factor because the AHP method is proved validate in practice although it may induce inner inconsistency. Suppose the obtained initial weight vector is $W$.

\subsubsection{Assessment Expression}

Since uncertain expressions are often used in a real situation, this paper uses linguistic terms to express assessments [11,23]. These linguistic terms are illustrated by fuzzy set. Moreover, the universe of these fuzzy set are unified to real interval $[0,1]$ which means the satisfactory degree of the agent to a particular attribute. Therefore, all fuzzy sets have same universe which is convenient for aggregating assessments.

Suppose $g_{k}(k=0,1, \ldots, K)$ is the satisfactory degree measure for attribute $c_{k}$. Then an agent's opinion on the goods in terms of attribute $c_{k}$ is denoted by $g_{k}(u)$ where $u\left(\in U_{k}\right)$ is the real attribute value of attribute $c_{k}$ and $U_{k}$ is the real universe for attribute $c_{k}$. For instance, departing time is an attribute for a flight ticket. The possible departing time in a day is from $0: 00$ to $23: 59$. For any time slot $u$, an agent may present a satisfactory degree such as departing at $7: 30$ is with satisfactory degree 0.9 and departing at $3: 00$ is with 0.3 .

In the following, let $A=\left\{a_{1}, \ldots, a_{n}\right\}$ be the set of used assessment terms which are fuzzy sets on satisfactory degree $[0,1]$. Then a numeric satisfactory degree is transformed to a linguistic term. Continue the above example, suppose the assessment set is as shown in Figure 2. Notice that $a_{7}$ is with the biggest the membership degree for 0.9 , the assessment for departing at $7: 30$ is $a_{6}$ by the maximum membership degree principle. Similarly, the assessment for 0.3 is $a_{2}$.

\subsubsection{Assessments Aggregation}

An aggregated assessment is the agent's overall opinion/preference on the goods in terms of multiple attributes. The change of an attribute value may leads to the alternation of an assessment. Instinct natures of different attributes increase the difficulty and uncertainty for obtaining an overall assessment. Notice that an agent's preference on an individual attribute can be expressed through the agent's satisfactory degree on that attribute. This paper uses an satisfactory degree measure as the common universe of assessment.

Based on assessment on each individual attribute, an overall assessment can be obtained as follows. Suppose the individual assessments of all attributes are $v_{0}, v_{1}, \ldots, v_{K}$ and the weights of

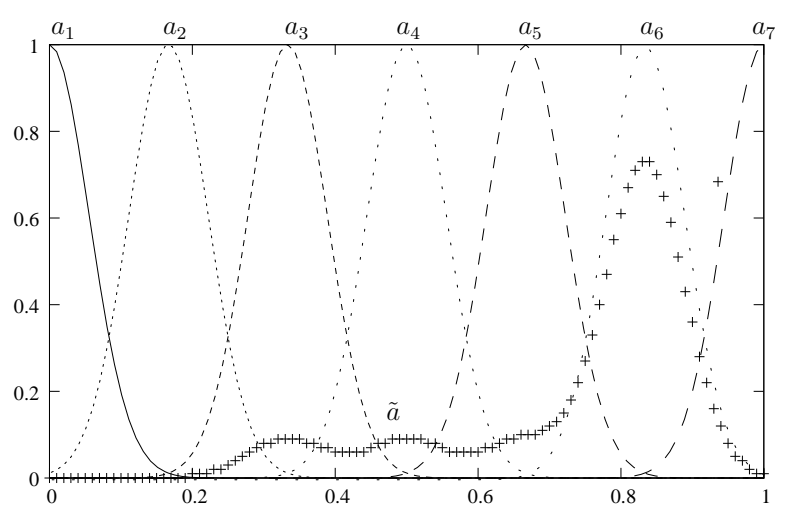

Figure 2: Assessment aggregation

them are $w_{0}, w_{1}, \ldots, w_{k}$ respectively. Then an overall assessment is obtained by

$$
a=\operatorname{Agg}\left\{\left(v_{0}, w_{0}\right),\left(v_{1}, w_{1}\right), \ldots,\left(v_{K}, w_{K}\right)\right\}
$$

where Agg is a selected aggregation method, $v_{k} \in A(k=0,1, \ldots, K)$ is the linguistic assessment on attribute $c_{k}$.

To get an overall assessment in terms of a set of criteria, an aggregation method Agg is applied. Some existing methods can be used here, such as OWA operator [24, 25], 2-tuples linguistic aggregation $[5,6,11]$, and Weighted-sum. For convenience, we use the weighted-sum-based method to obtain an overall assessment as follows.

First, we construct a fuzzy set $\tilde{a}$ on $[0,1]$ through

$$
\tilde{a}(u)=\sum_{k=0}^{K} w_{k} \cdot v_{k}(u), \quad u \in[0,1],
$$

where $v_{k}(u)$ is the membership degree of $u$ in $v_{k}$.

Next, we calculate the distance between $\tilde{a}$ and $a_{i} \in A$ by

$$
d\left(\tilde{a}, a_{i}\right)=\int_{0}^{1}\left|\tilde{a}_{\lambda}-a_{i_{\lambda}}\right| \mathrm{d} \lambda .
$$

Finally, we select the nearest term(s) $a$ to $\tilde{a}$ as the overall assessment.

For example, $A$ has seven terms, namely, $a_{1}, a_{2}, \cdots, a_{7}$ as shown in Fig. 2. Suppose $\tilde{a}$ is the obtained fuzzy set. By comparing the distances between $\tilde{a}$ and each element in $A$, we know $a_{6}$ is the nearest item to $\tilde{a}$. Hence, $a_{6}$ will be taken as the overall assessment.

\subsection{Attitude Estimation}

Attitude is a learned predisposition to respond in a consistently favorable or unfavorable manner with respect to a given object [8]. In other words, the attitude is a preparation in advance of the actual response, constitutes an important determinant of the ensuing behavior. In AI, the fundamental notions to generate the desirable behaviors of the agents often include goals, beliefs, intentions, and commitments. Goal is a subset of states, and belief is a proposition that is held as true by an agent. Bratman [2] addresses the problem of defining the nature of intentions. Crucial to his argument is the subtle distinction between doing something intentionally and intending to do something. Cohen and Levesque [4], on the other hand, developed a logic in which intention is defined as a commitment to act in a certain mental state of believing throughout what he is doing. Thus to provide a definition of attitude that is concrete enough for computational purposes, we model attitude using goals, 
beliefs, intentions and commitments. From the Fishbein's [8] definition it is clear that when an attitude is adopted, an agent has to exhibit an appropriate behavior (predisposition means behave in a particular way). The exhibited behavior is based on a number of factors which depends on the nature of the dynamic world. Once an agent chose to adopt an attitude, it strives to maintain this attitude, until it reaches a situation where the agent may choose to drop its current attitude towards the object and adopt a new attitude towards the same object. Thus, an agent's attitude towards an object refers its persistent degree of commitment towards achieving one or several goals associated with the object, which give rise to an overall favorable or unfavorable behavior with regard to that object.

After conducting new assessment on the goods according to current price $p_{c}$, estimation of agent's attitude is implemented. In order to do so, the relationship between attitude and assessments is required. In general, the better the assessment on the given goods is, the stronger the attitude of bidding for that goods will be. However, this is by no means the unique relationship between attitude and assessment. For instance, other agents' competitive bidding sometimes can also cause strong willingness.

Suppose $E=\left\{e_{1}, \ldots, e_{m}\right\}$ is the set of attitude expressions, $A=\left\{a_{1}, \ldots, a_{n}\right\}$ is the set of assessments. Let

$$
r: \quad\left(a_{i} \Rightarrow e_{j}, \alpha_{i j}\right),
$$

be a given rule from an agent where $a_{i} \in A, e_{j} \in E$, and $\alpha_{i j}$ is the reliability degree of the rule. Such rule depicts the approximate degree of an agent's attitude $e_{j}$ to which the agent can win the bid under the assumption that the overall assessment is $a_{i}$. Furthermore, these rules can be treated as a set of fuzzy sets on $A$ such that the membership degree in a fuzzy set $f_{j}$ corresponding to eagerness $e_{j}$ is $\alpha_{i j}$. Obviously, $f_{j}$ is an integration of rules $\left(a_{i} \Rightarrow e_{j}, \alpha_{i j}\right)$ $(i=1, \ldots, n)$, which is able to be treated as an alias of $e_{j}$. Hence, the fuzzy set $f_{j}$ is also called attitude in the following without other specification.

Based on the rules in $R$, an agent can estimate the possible attitude of the agent when it learns the current overall assessment. A set of fuzzy sets is obtained through the following way: suppose the overall assessment is $a_{c}$, then the attitude at the moment is determined by the maximum membership degree principle

$$
e_{c} \in E\left(a_{c}\right)=\left\{e_{j} \in E \mid f_{j}\left(a_{c}\right) \geqslant f_{i}\left(a_{c}\right) \text { if } i \neq j\right\} .
$$

Notice that such determined $e_{c}$ may not necessarily be unique. In the following, we call $E\left(a_{c}\right)$ the candidate attitude set under $a_{c}$.

Once the current attitude of the agent is determine, requirements for search new bids can then be determined. The main requirements include identifying required overall assessment and finding the candidate prices.

\subsection{Overall Assessment}

Prerequisite of overall assessment is the basic requirement on the goods such that the agent has the highest possibility to win a bid under the current attitude. To find the prerequisite of overall assessment, an order is firstly defined in $E$ according to the strength of attitude. Without loss of generality, suppose $e_{i}<e_{j}$ if $i<j$. Therefore, it is possible to select the strongest element from $E\left(a_{c}\right)$. Then the strongest element in $E\left(a_{c}\right)$ is chosen as the first candidate attitude to determine the prerequisite of overall assessment.

Suppose a set of rules $\bar{R}$ is determined such that any $\bar{r} \in \bar{R}$ is of form

$$
\bar{r}: \quad\left(e_{j} \Rightarrow a_{i}, \bar{\alpha}_{i j}\right),
$$

where $e_{j} \in E, a_{i} \in A$, and $\bar{\alpha}_{i j}$ is the reliability degree. These rules indicate to what extend an assessment $a_{i}$ is obtained given an attitude $e_{j}$.

Based on the maximum membership degree principle, a set of candidate assessment is determined such that

$$
A\left(e_{c}\right)=\left\{a_{i} \in A \mid \bar{f}_{i}\left(e_{c}\right) \geqslant \bar{f}_{j}\left(e_{c}\right) \text { if } i \neq j\right\},
$$

where $\bar{f}_{i}$ is the counterpart to $f_{i}$. Each element $a$ in $A\left(e_{c}\right)$ is called a candidate assessment under eagerness $e_{c}$.

\subsection{Agent Price Determination}

An agent's assessment demonstrates some expectation on the quality of the goods. As other criteria except the price are seldom changeable in an auction, this is regarded in terms of price.

Suppose $U_{0}=\left[p_{l}, p_{u}\right]$ is the real range of price. A price range $U(a)$ corresponding to a candidate assessment $a$ is a subset of $U_{0}$ such that for any $u \in U(a)$, the assessment based on $u$ and $W$ is $a$. Notice that an assessment is a fuzzy set on the satisfactory degree $[0,1]$ which is the bridge between assessment and price, a price range is determined by the following steps.

Step 1: We divide the satisfactory degree $[0,1]$ into $n$ subsets $D_{1}, D_{2}, \ldots, D_{n}$ such that

$$
a_{i}(d) \geqslant a_{j}(d)
$$

for any $d \in D_{i}$ and $j \neq i$, i.e., element in $D_{i}$ with biggest membership degree in $a_{i}$.

Step 2: For $D_{a}$ corresponding to a candidate assessment $a$, we select price in $U_{0}$ such that $g_{a}(u) \in D_{a}$. $U_{a}$ is called a candidate bid set. Concerning that the satisfactory degree is continuously change with the price, we assume that $U_{a}$ is an interval in $U_{0}$. Hence, let $p_{l a}$ and $p_{u a}$ be the left and right boundary of $U_{a}$.

Thus, a candidate price range for assessment $a$ is determined. For instance, Fig. 3 indicates that the price range corresponding to assessment $a_{6}$ is $\$[900-1000]$.

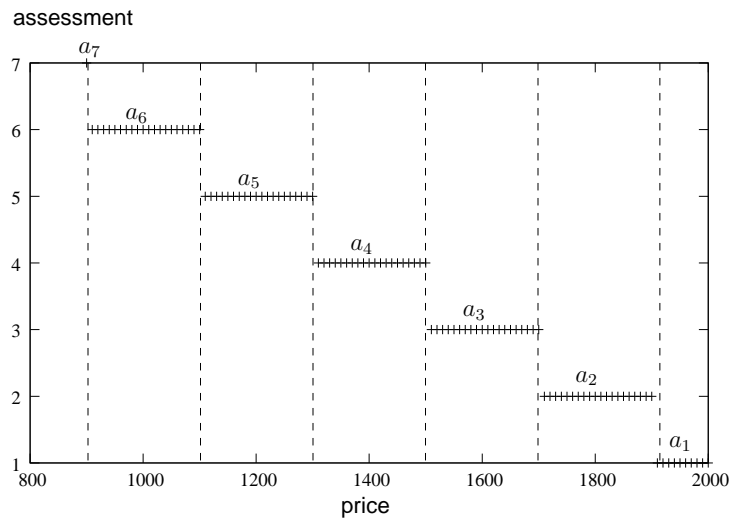

Figure 3: Candidate price range.

Suppose for any element in $A\left(e_{c}\right)$, we have obtained a corresponding candidate price range. Because new bid should higher than the present price $p_{c}$, a candidate price set for $A\left(e_{c}\right)$ is determined by

$$
\begin{aligned}
U_{A}= & \left\{p_{l i} \mid p_{l i}>p_{c}, a_{i} \in A\left(e_{c}\right)\right\} \\
& \cup\left\{p_{u i} \mid p_{u i}>p_{c}, a_{i} \in A\left(e_{c}\right)\right\} .
\end{aligned}
$$

As it can be seen that the candidate price range may not exist under some assessments, in these case, a weaker attitude is selected to repeat the candidate price determination process until a range is found or the attitude is weaker than an acceptable level. 
Suppose $U_{A}$ is a found price range, there must be a smallest element $b$ in it. Then $b$ is selected as the new bid. For example, in Fig. 3 , suppose the current flight price is $\$ 900$, the prerequisite assessment is $a_{6}$, and the least increment is $\$ 50$, then the new should be $\$ 950$.

\section{EXPERIMENT EVALUATION}

In this section, an experiment implements the fuzzy bidding strategy in a scenario in which an agent intends to book flight tickets. Six factors (as shown in Table 1) are concerned in this situation, i.e. ticket price $\left(c_{0}\right)$, depart time $\left(c_{1}\right)$, arrival time $\left(c_{2}\right)$, number of stops $\left(c_{3}\right)$, seat positions $\left(c_{4}\right)$, and travel season $\left(c_{5}\right)$. The flight ticket bid for is a return ticket to destination $D$ with the following properties:

- price: $\$ 800$ - $\$ 2000$;

- depart time: 18:00 PM, Wednesday;

- return arrival time: 10:00 AM, Friday;

- number of stops: 1 ;

- seat position: window;

- travel season: April (off-peak season).

Table 1: Concerned attributes of a flight ticket.

\begin{tabular}{lclr}
\hline Attributes & Symb. & Values range & Weights \\
\hline price & $c_{0}$ & $\$[800-2000]$ & 0.4 \\
depart time & $c_{1}$ & Sun. 0:00 - Sat. 24:00 & 0.1 \\
arrival time & $c_{2}$ & Sun. 0:00 - Sat. 24:00 & 0.1 \\
stops & $c_{3}$ & 0, 1, 2, 3 & 0.1 \\
seat position & $c_{4}$ & window, aisle, middle & 0.1 \\
flight season & $c_{5}$ & Jan. 01 - Dec. 31 & 0.2 \\
\hline
\end{tabular}

Suppose the identified perspective of an agent is summarized as below:

- The agent prefers to a cheaper ticket and agrees to that the cheaper the better.

- The agent prefers to travel at the weekend rather than at working day.

- The agent prefers to no stop travel.

- The agent prefers to aisle seat then window seat.

- The agent prefers to travel during off-peak season rather than peak season.

- The agent thinks the flight price is the most important factor, secondly the travel season, and other factors are of same importance.

Based on the agent's perspective, the agent evaluates the ticket using seven terms (shown in Figure 4$)$, i.e., very bad $\left(a_{1}\right)$, bad $\left(a_{2}\right)$, slightly bad $\left(a_{3}\right)$, acceptable $\left(a_{4}\right)$, fairly good $\left(a_{5}\right)$, good $\left(a_{6}\right)$, and very good $\left(a_{7}\right)$. The seven terms are expressed by fuzzy sets on the satisfactory degree $[0,1]$ as below (see Figure 4):

$$
f_{a_{i}}=e^{-162\left(x-(i-1) \frac{1}{6}\right)^{2}}, \quad i=1, \ldots, 7 .
$$

The assessment on each individual factor is

\begin{tabular}{cl}
\hline Attribute & Assessment \\
\hline$c_{0}$ & (no assessment) \\
$c_{1}$ & good $\left(a_{6}\right)$ \\
$c_{2}$ & fairly good $\left(a_{5}\right)$ \\
$c_{3}$ & slightly bad $\left(a_{3}\right)$ \\
$c_{4}$ & acceptable $\left(a_{4}\right)$ \\
$c_{5}$ & good $\left(a_{6}\right)$ \\
\hline
\end{tabular}

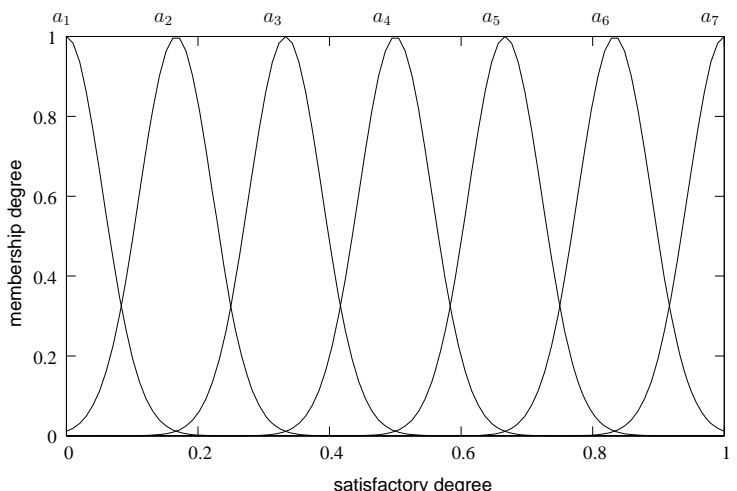

Figure 4: Assessment terms.

As the ticket price is the changeable factor, the assessment on it is determined dynamically. For convenience, suppose the agent's satisfactory degree measure on price is expressed by a linear function as below:

$$
g_{0}(p)=\frac{2000-p}{1200} .
$$

Now assume the current price $\left(p_{c}\right)$ is $\$ 900$, the agent is required to determine a new bid in this situation.

First, the satisfactory degree of the current price is calculated by Eq. (10), which is 0.91. Because $f_{a_{7}}(0.91)=0.35$ and $f_{a_{6}}(0.92)=$ 0.82 , the assessment for $p_{c}$ is $a_{6}$ (good).

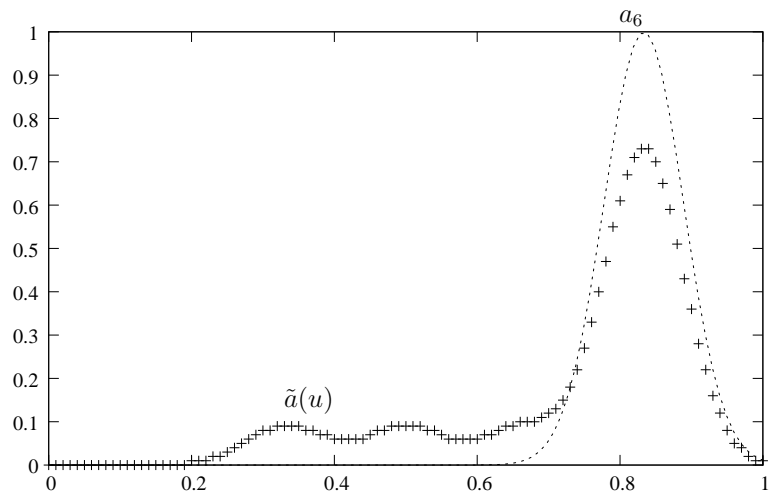

Figure 5: Overall assessment.

Next, since the price changes will affect the weights of all factors, a new overall assessment of the ticket is calculated. Suppose the increase of price weight is 0.05 , i.e., the current weight of price is $w_{0}^{\prime}=0.45$. Then the weights of other factors are calculated by Eq. (??), and they are

$$
\begin{aligned}
w_{1}^{\prime}=w_{2}^{\prime}=w_{3}^{\prime}=w_{4}^{\prime}= & 0.09 \\
w_{5}^{\prime}= & 0.19 .
\end{aligned}
$$


Therefore, a fuzzy set $\tilde{a}(u)$ is obtained $(\tilde{a}(u)$ in Figure 5$)$. Then by Eq. (3), the most nearest assessment to $\tilde{a}$ is $a_{6}$. So the new overall assessment for the ticket is $a_{6}$.

Table 2: Rule set for attitude estimation.

\begin{tabular}{c|ccccc}
\hline \multirow{2}{*}{ ass. } & \multicolumn{5}{|c}{ attitude } \\
\cline { 2 - 6 }$a_{1}$ & $e_{1}$ & $e_{2}$ & $e_{3}$ & $e_{4}$ & $e_{5}$ \\
$a_{2}$ & 0.17 & 0.23 & 0.20 & 0.27 & 0.13 \\
$a_{3}$ & 0.10 & 0.28 & 0.22 & 0.26 & 0.13 \\
$a_{4}$ & 0.17 & 0.26 & 0.18 & 0.32 & 0.13 \\
$a_{5}$ & 0.12 & 0.25 & 0.27 & 0.23 & 0.12 \\
$a_{6}$ & 0.12 & 0.26 & 0.26 & 0.23 & 0.16 \\
$a_{7}$ & 0.12 & 0.24 & 0.31 & 0.24 & 0.13 \\
\hline
\end{tabular}

Then the agent needs to estimate the agent's attitude according to this assessment. Suppose the agent uses five terms to distinguish the attitude, i.e., none $\left(e_{1}\right)$, slightly $\left(e_{2}\right), \operatorname{medium}\left(e_{3}\right)$, strong $\left(e_{4}\right)$, and very strong $\left(e_{5}\right)$. In order to estimate the agent's attitude, a set of rules of form Eq. (4) are extracted from a historical auction records, which are illustrated in Table 2 and Figure 6.

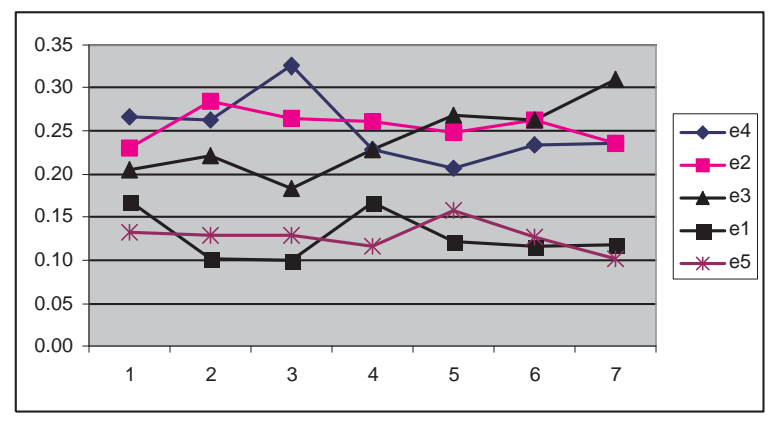

Figure 6: Illustration for rule set $R$.

Table 3: Rule set for prerequisite of assessment identification.

\begin{tabular}{c|ccccccc}
\hline & \multicolumn{7}{|c}{ assessment } \\
\cline { 2 - 8 } att. & $a_{1}$ & $a_{2}$ & $a_{3}$ & $a_{4}$ & $a_{5}$ & $a_{6}$ & $a_{7}$ \\
\hline$e_{1}$ & 0.11 & 0.16 & 0.12 & 0.24 & 0.16 & 0.16 & 0.06 \\
$e_{2}$ & 0.07 & 0.21 & 0.15 & 0.18 & 0.16 & 0.17 & 0.06 \\
$e_{3}$ & 0.07 & 0.18 & 0.11 & 0.17 & 0.18 & 0.19 & 0.09 \\
$e_{4}$ & 0.09 & 0.20 & 0.19 & 0.16 & 0.13 & 0.16 & 0.06 \\
$e_{5}$ & 0.08 & 0.19 & 0.15 & 0.16 & 0.20 & 0.17 & 0.05 \\
\hline
\end{tabular}

By Figure 6, the agent's attitudes at this moment are $e_{2}$ and $e_{3}$ because they have the highest reliability. Because $e_{3}$ is stronger than $e_{2}$, the agent first searches possible bids under the attitude $e_{3}$. Based on $e_{3}$, the agent discovers that $a_{6}$ is the most preferred assessment on the ticket through rules in Table 3. Hence, it will determine a candidate price range based on the assessment $a_{6}$.

Based on Figure 4, the agent can divide the satisfactory degree interval $[0,1]$ into seven sub-intervals. In this figure, the interval corresponding to assessment $a_{6}$ is $\$[900-1100]$ and the current price $p_{c}$ belongs to this interval. Hence, a new bid can be selected from the interval. According to the FAB-strategy, the smallest one greater than the $p_{c}(900)$ will be selected. For instance, if the least increase is $\$ 50$, then the new bid $b$ is $\$ 950$.

\section{CONCLUSIONS}

In this paper, a novel fuzzy bidding strategy (FAA-Bid) is discussed. It was noticed that agents, which adopt attitudes, behave more flexibly and efficiently than agents without attitude and adapt more easily to dynamic situations. Another unique idea presented in this paper is that to deal quantitatively the imprecision or uncertainty of multiple attributes of items to acquire in auctions, fuzzy set technique is used. The fuzzy logic provides attitude based agents provide resources in the decision making process of bidding agent. The bidding strategy also allows for flexible heuristics both for the overall gain and for individual attribute evaluations. It also explores the relationships between evaluations of different attributes using Analytic Hierarchy Process method [22].

There are a number of areas of further investigation. In future we would further like to explore the development of strategies for multiple auctions. We would also like to compare our bidding techniques with other decision theoretic approaches to determine the relative strengths and weaknesses of these methods. Different strategies may perform well in some environments but may perform poorly in another. The numbers of strategies that can be employed are endless and the search space is huge. To address this issue, we intend to use learning techniques to obtain a model of the price dynamics based on the past data and to search for most successful strategies in predefined environments in an offline fashion.

\section{REFERENCES}

[1] P. Anthony and N.R.Jennings. Evolving bidding strategies for multiple auctions. In Proceedings of 15th European Conference on Artificial Intelligence, pages 187-192, Netherlands, 2002.

[2] M. E. Bratman. Intentions, Plans and Practical Reason. Harvard University Press, Cambridge, MA, 1987.

[3] A. Byde, C. Priest, and N.R.Jennings. Decision procedures for multiple auctions. In Proceedings of the First International Joint Conference on Autonomous agents and Multiagent Systems, pages 613-620, Bologana, Italy, July 2002.

[4] P. R. Cohen and H. J. Levesque. Teamwork. Cognitive Science and Artificial Intelligence, 25(4), 1991.

[5] M. Delgado, F. Herrera, and E. Herrera-Viedma. Combining linguistic information in a distributed intelligent agent model for information gathering on the internet. In P. Wang, editor, Computing with words, pages 251-276. John Wiley and Sons, Inc., 2001.

[6] M. Delgado, F. Herrera, E. Herrera-Viedma, J. L. Verdegay, and M. A. Vila. Aggregation of linguistic information based on a symbolic approach. In L. Zadeh and J. Kacpryzk, editors, Computing with Words in Information/Intelligent Systems I. Foundations, pages 428-440. Physica-Verlag, 1999.

[7] P. Faratin, C. Sierra, and N. Jennings. Negotiation decision functions for autonomous agents. International Journal of Robotics and Autonomous Systems, 24(3-4):159-185, 1998.

[8] M. Fishbein and I. Ajzen. Belief, Attitude, Intention and Behaviour: An Introduction to theory and research. Addison-Wesley, Reading, MA, USA, 1975.

[9] A. Greenwald and P. Stone. Autonomous bidding agents in the trading agent competition. IEEE Internet Computing, pages 52-60, March-April 2001.

[10] M. He, H. F. Leung, and N. R. Jennings. A fuzzy-logic based bidding strategy for autonomous agents in continuous double 
auctions. IEEE Transactions on Knowledge and data Engineering, 15(6):1345-1363, 2003.

[11] F. Herrera, E. Herrera-Viedma, and F. Chiclana. Multiperson decision-making based on multiplicative preference relations. European J. Operational Research, 129:372-385, 2001.

[12] N. Jennings, P. Faratin, A. Lomuscio, S. Parsons, C. Sierra, and M. Wooldrige. Automated negotiation: prospects, methods and challenges. Group Decision and Negotiation, 10(2):199-215, 2001.

[13] R. Kowalcyzk. On negotiation as a distributed fuzzy constraint satisfaction problem. In Proceedings DEXA e-Negotiation Workshop, pages 631-637, 2000.

[14] R. Kowalcyzk and V. Bui. On fuzzy e-negotiation agents: Autonomous negotiation with incomplete and imprecise information. In Proceedings Dexa e-Negotiation Workshop, 2000.

[15] X. Luo, N. Jennings, N. Shadbolt, H. Leung, and J. Lee. A fuzzy constraint based model for bilateral, multi-issue negotiation in semi-competitive environments. Artificial Intelligence, 148(1-2):53-102, 2003.

[16] X. Luo, C. Zhang, and N. Jennings. A hybrid model of sharing between fuzzy, uncertain and default reasoning models in multi-agent systems. International Journal of Uncertainty, Fuzziness Knowledge Based Systems, 10(4):401-450, 2002.

[17] H. Ma and H.-F. Leung. An adaptive attitude bidding strategy for agents in continuous double auctions. Electronic Commerce Research and Applications, 6:383-398, 2007.

[18] N. Matos and C. Sierra. Evolutionary computing and negotiating agents. In Agent Mediated Electronic Commerce, volume 1571 of Lecture Notes in Artificial Intelligence, pages 126-150. Springer-Verlag, New York, 1998.

[19] P.Anthony and N.R.Jennings. Developing a bidding agent for multiple heterogeneous auctions. ACM transactions on Internet Technology, 3(3):185-217, 2003.

[20] C. Priest, C. Bartolini, and I.Philips. Algorithm design for agents which participate in multiple simultaneous auctions. In In Agent Mediated Electronic Commerce III (LNAI), pages 139-154. Berlin, German, 2001.

[21] P.Stone, M. Littman, S.Singh, and M.Kearns. Attac-2000: An adaptive autonomous bidding agent. Journal of Artificial Intelligence Research, 15:189-206, 2001.

[22] T. Saaty. The Analytic Hierarchy Process. McGraw Hill, NY, 1980.

[23] R. R. Yager. On ordered weighted averaging aggregation operators in multicriteria decisionmaking. 18(1):183-190, 1988.

[24] R. R. Yager. Families of OWA operators. 59:125-148, 1993.

[25] R. R. Yager. OWA aggregation over a continuous interval argument with applications to decision making. IEEE Transactions on Systems, Man, and Cybernetics-Part B: Cybernetics, 34(5):1952-1963, 2004.

[26] D. Zeng and K. Sycara. Bayesian learning in negotiation. International Journal Human Computer Studies, 48:125-141, 1998. 